\title{
Satisfacción del usuario de turismo náutico en la actividad charter a vela y motor como propuesta alternativa
}

\section{Satisfaction of the user of nautical tourism in the activity charter to sail and engine as alternative proposal}

\author{
Vicente Morales Baños, Francisco José Borrego Balsalobre* y Salvador Angosto Sánchez*
}

Facultad de Ciencias del Deporte San Javier (Universidad de Murcia). Campus de Excelencia Internacional Regional “Campus Mare Nostrum”.

\begin{abstract}
Resumen: El objetivo de este trabajo fue analizar la calidad de los servicios chárter náuticos en las islas baleares mediante un cuestionario previamente validado que dimensiona cuatro factores de medición. Se encuestaron 185 usuarios de diferentes nacionalidades, edades y género, usuarios de distintas empresas dedicadas al sector entre los meses de Julio y Agosto. Los resultados mostraron que no había diferencias significativas por grupos de edad para cada uno de los factores pero que el que en términos globales el que más importancia mostraba era el de satisfacción. Del mismo modo la satisfacción y el valor percibido mostraban relación directa con las intenciones de práctica futuras.

Palabras clave: Servicio náutico, Turismo, Satisfacción, Intención futura, Chárter náutico.
\end{abstract}

Abstract: The aim of this work was to analyze the quality of nautical charter services in the Balearic Islands by means of a previously validated questionnaire that measures four measurement factors. We surveyed 185 users of different nationalities, ages and gender, users of different companies dedicated to the sector between July and August. The results showed that there were no significant differences by age groups for each of the factors, but that in general the most important was satisfaction. Likewise satisfaction and perceived value were directly related to future practice intentions.

Key words: Nautical service, Tourism, Satisfaction, Future intention, Nautical charter.

\section{Introducción}

Para hacer referencia al concepto de turismo náutico previamente habría que hacerlo desde el punto de vista general y, territorialmente, a continuación situar en el marco internacional a los países que, como España, son consumidores de este tipo de turismo. Se define turismo como un conjunto de relaciones que surgen como consecuencia de un viaje, siempre y cuando éste no dure más de un año. Es aquí donde entran en juego los servicios que el usuario desea adquirir a lo largo del mismo, así como los medios y recursos que el destino muestra para ello (Wang, 1999). Pero, aunque este es un fenómeno que va "in crescendo" a nivel mundial, debido en parte a la globalización en la actualidad, es importante remarcar lo que supone el mismo para la península ibérica y sus cabildos insulares; donde las condiciones climatológicas y la ley de oferta y demanda lo sitúan como uno de los destinos turísticos líderes en el globo terráqueo (Lepp \& Gibson, 2008). Sin ir más lejos, según la Organización Mundial de Turismo, en el ejercicio de 2016 visitaron España 75.3 millones de turistas; ingresando un $8 \%$ más de lo que se hizo en 2015.

Esto, pone de manifiesto también las crecientes aportaciones académicas al estudio del sector actualmente; en gran medida debido a la cantidad de ingresos económicos que

Dirección para correspondencia [Correspodence address]: Francisco José Borrego Balsalobre. Facultad Ciencias del Deporte, Av. Argentina s/n, San Javier, Murcia (Espańa). E-mail: franborrego@um.es supone para los países poder resultar atractivos al consumidor de viajes y el gran interés que suscita como consecuencia evidente de ello. En este sentido, el presidente y director ejecutivo de la asociación empresarial World Travel \& Tourism Council, David Scowsill, indicó el pasado ańo que: "a pesar de la incertidumbre en la economía internacional y de los retos específicos para el viaje y el turismo del 2015, el sector creció en un $3.7 \%$, contribuyendo a un total del $9.8 \%$ del PIB global"; suponiendo en Espańa el 11.1\% del total de la economía española.

$\mathrm{Y}$ es precisamente dentro de este marco de sectores que viven del turismo y lo nutren al mismo tiempo en completa armonía donde cobra especial importancia la actividad física y el deporte. Tanto es así, que en la actualidad se observa un gran desarrollo del sector deportivo dentro del turismo. Crecimiento este, que se explica con el cambio de estatus que el deporte ha tenido en la sociedad, pasando de ser considerado una forma de ocupar el tiempo libre a considerarse como un indicador de bienestar social y calidad de vida (Getz, 2008; López, Díaz, Radzimiński, \& Jastrzębski, 2017; Vespalec, Pavlík, Zvonař, \& Zeman, 2016).

Por ello, una de las áreas de estudio de la gestión del deporte que está adquiriendo gran protagonismo es la del análisis de la satisfacción de los usuarios y la calidad de servicio percibido (Shonk \& Chelladurai, 2008). En la actualidad, la calidad se ha convertido en una necesidad para garantizar la 
continuidad y el progreso de las empresas, generando beneficios que repercutan en los clientes, directivos, empleados e imagen de la organización. Además, el gran desarrollo que en los últimos años ha tenido el sector servicios, ha hecho que se afronte la calidad desde el punto de vista de calidad de servicio, considerándose éste como el mayor potencial en cuanto a superioridad competitiva que hoy día pueden tener las empresas (Moon, Kim, Ko, Connaughton, \& Lee, 2011).

Todo esto justifica el aumento de las investigaciones que pretenden identificar los puntos clave para obtener el máximo rendimiento, coincidiendo una parte de ellas en el estudio de la satisfacción y la calidad de los servicios deportivos desde el punto de vista del usuario. La principal aportación en el estudio de la calidad de servicio es la realizada por Parasuraman, Zeithaml, \& Berry (1985). Estos autores, desarrollan un modelo sobre la calidad de servicio percibida con cinco desajustes que a su entender son la causa de la calidad o la no calidad. El desajuste cinco, definido como la diferencia entre el servicio esperado y el servicio percibido, es el concepto clave del modelo y viene determinado por otros cuatro desajustes que ocurren en los procesos propios de las empresas. Estos desajustes tienen su origen en la falta de conocimiento de las expectativas del usuario.

Proveniente de esta primera aproximación, Parasuraman, Zeithaml, \& Berry (1988) definen la calidad de servicio percibida como "un juicio global, o actitud, relativa a la superioridad del servicio", y aplicando el concepto al deporte, la calidad percibida de servicios deportivos puede ser una medida de los juicios acerca de la excelencia global del prestatario al dar un servicio al consumidor de deporte, o "la satisfacción de los requerimientos, deseos y expectativas de los clientesusuarios de un servicio deportivo" (Calabuig-Moreno et al., 2016).

\section{El sector náutico deportivo como apuesta turística}

La organización del viaje, el tipo de actividades a realizar o la motivaciones del turista pueden generar diversas clases de turismo, entre las que se encuentra el turismo de sol y playa, que aunque principalmente se centra en la época estival, es uno de los más atractivos y demandados cobrando especial importancia en países que dan su cara al mar por la cantidad de empleo directo e indirecto que genera. (Yepes \& Medina, 2005). Además este es uno de los tipos de turismo que permiten fusionarse con otros como el cultural, el deportivo, el de salud o incluso el de negocios.

El marketing, como elemento fundamental en el desarrollo de estrategias en las empresas que ofertan servicios, no es ajeno a estos cambios y también ha evolucionado con la finalidad de dar respuestas adecuadas a las nuevas necesidades emergentes para adaptarse así a las necesidades y demandas de los clientes. Hemos pasado de una concepción inicial, ba- sada en la producción, a otra centrada en el propio cliente, basada fundamentalmente en principios transaccionales y relacionales, para orientarse, a partir de los últimos desarrollos enunciados, a los valores, incorporando elementos vinculados a las emociones y los sentimientos (Robinot \& Giannelloni, 2010).

En un sector como el turístico, donde la interacción entre clientes y prestadores de servicios es elevada, los aspectos psicológicos adquieren una especial relevancia y procede analizar detalladamente las sensaciones, impresiones y percepciones de los clientes con la finalidad de conocer qué factores y de qué manera influyen en variables como el valor percibido, la calidad, la satisfacción y, en último término, en la lealtad de los consumidores. El turismo náutico, a pesar de su juventud, ha ido ganando protagonismo a lo largo de los últimos años en este sentido en el conjunto del sector turístico, ofreciendo una posibilidad más de diversificación que puede contribuir a atomizar una oferta excesivamente centrada en el segmento de "sol y playa". El desarrollo de este tipo de productos específicos responde al incremento del grado de heterogeneidad que presenta la propia demanda y que refleja unas preferencias y necesidades específicas que conviene satisfacer de un modo eficiente y adecuado (Favro, Grzetic, \& Kovacic, 2010; Kovacic, 2010).

La escasa atención recibida por parte del turismo náutico en el ámbito de la investigación en marketing implica que sean muchas las cuestiones a abordar, aunque basándonos en la evolución experimentada por el concepto de marketing en los últimos años, el estudio del comportamiento del consumidor se revela como uno de los más importantes campos que pueden ser objeto de interés (Jovanovic, Dragin, Armenski, Pavic, \& Davidovic, 2013; Mikulic, Kresic, \& Kozic, 2015). Se puede entender dentro de este comportamiento la calidad general, el valor percibido, la satisfacción y las intenciones futuras de práctica (Angosto, 2014).

\section{Método}

\section{Características de la muestra}

La muestra estuvo compuesta por un total 185 usuarios de servicios náuticos de chárter a vela y motor de las islas baleares. El sexo de los participantes fue un $59,5 \%$ hombres y un $40,5 \%$ mujeres. Respecto a la edad, el $37,3 \%$ eran menores de 30 ańos, un 31,9\% entre 30 y 45 ańos y los mayores de 45 años fueron $30,8 \%$. Los países de procedencia fueron: España $(24,3 \%)$, Alemania $(16,2 \%)$, Inglaterra $(15,7 \%)$, Francia y Argentina (7,6\%), USA (5,9\%), Italia (4,9\%), Turquía y Gales (3,8\%), Dinamarca (3,2\%), Holanda (2,2\%), Méjico $(1,6 \%)$ y Australia, Bélgica, Venezuela $(1,1)$. 


\section{Diseño y variables objeto de estudio}

Se trató de un diseño cuantitativo no experimental, transversal y descriptivo, a través de encuestas por muestreo para conocer los niveles de intenciones futuras de práctica y realización de actividades náuticas para embarcaciones a motor y a vela de esloras entre los 20 y 50 pies.

\section{Procedimiento y materiales empleados}

\section{Procedimiento}

Se administró un cuestionario de forma telemática, para el cual se generó un formulario online mediante la herramienta Google Drive. Se solicitó permiso previo a la empresa. Los usuarios realizaban el cuestionario online, una vez había finalizado el servicio náutico contratado. Los datos se recogieron entre los meses de Julio y Agosto de 2016.

\section{Instrumento}

El cuestionario utilizado ha sido la adaptación de una escala validada por Angosto (2014) compuesta por un total de 14 ítems divididos en cuatro dimensiones: calidad general del evento, valor percibido e intenciones futuras a la que se ańadieron unos ítems sobre la satisfacción general con el evento (Tabla 1). La alternativa de respuesta ha sido escala tipo Likert de 6 anclajes (1- Muy en desacuerdo y 6 - Muy de acuerdo). La fiabilidad total de la escala presentó un alto valor de alfa de Cronbach de 0,971.

Tabla 1. Descriptivo del instrumento y fiabilidad de cada escala.

\begin{tabular}{cccc}
\hline Dimensión & No Items & Adaptación & Alfa de Cronbach \\
\hline Calidad General & 4 & Hightower, Brady \& Baker (2002) &, 906 \\
Valor Percibido & 3 & Gallarza y Gil (2006), Hightower, et al. (2002) &, 906 \\
Intenciones Futuras & 3 & Zeithmal, Berry \& Parasunaman (1996) &, 944 \\
Satisfacción & 4 & Crespo (2011), Oliver (1981) &, 976 \\
\hline
\end{tabular}

Análisis de datos

El análisis de los datos se realizó, con el paquete estadístico SPPS 19 con licencia de la Universidad de Murcia. Las pruebas que se hicieron, fue análisis descriptivo y una ANOVA entre grupos, una regresión lineal y el Alfa de Cronbach para calcular la fiabilidad del instrumento.

\section{Resultados}

Los resultados (Tabla 2) indicaron que a nivel general el factor que obtuvo una mejor valoración fue la satisfacción general con el servicio tenido por parte de los usuarios de los servicios náuticos con un valor de 5,11 , seguido con una puntuación superior también a 5 puntos las intenciones futuras de volver a utilizar el servicio y recomendarlo. La calidad general arrojó un valor bastante alto de 4,89 puntos, siendo el valor percibido el factor menos valorado por los usuarios con 4,64 puntos.

En cuanto a los resultados atendiendo a la edad de los usuarios, los clientes menores de 30 años fueron aquellos que tuvieron una mejor valoración en todas las escalas con puntuaciones superiores a cinco puntos en todos los factores excepto el valor percibido (4,82 puntos). Los usuarios de mediana edad (de 30 a 45 años), presentaron mejor valoración en calidad general ( 4,82 puntos) y satisfacción $(5,11$ puntos) que los usuarios de más de 45 años, quienes presentaron una mejor puntuación para los factores intenciones futuras y valor percibido (5,00 y 4,66 puntos respectivamente).

Tabla 2. Análisis descriptivo de las escalas en general y por edad (M[DT]) y prueba ANOVA.

\begin{tabular}{ccccccc}
\hline Dimensión & Total & Menos de 30 ańos & Entre 30 y 45 ańos & Más de 45 años & $\mathrm{F}$ & Sig. \\
\hline Calidad general & $4,89[ \pm, 85]$ & $5,09[ \pm, 83]$ & $4,82[ \pm, 82]$ & $4,78[ \pm, 90]$ & 2,31 &, 101 \\
Valor percibido & $4,64[ \pm, 93]$ & $4,82[ \pm, 99]$ & $4,47[ \pm, 84]$ & $4,66[ \pm, 95]$ & 2,24 &, 109 \\
Intenciones futuras & $5,05[ \pm, 88]$ & $5,20[ \pm, 89]$ & $4,97[ \pm, 91]$ & $5,00[ \pm, 84]$ & 1,31 &, 272 \\
Satisfacción & $5,11[ \pm, 89]$ & $5,29[ \pm, 86]$ & $5,11[ \pm, 82]$ & $4,93[ \pm, 97]$ & 2,42 &, 092 \\
\hline
\end{tabular}

La prueba ANOVA no arrojó diferencias significativas entre grupos. Se obtuvo una alta tendencia a la significación en la satisfacción con una $\mathrm{p}=, 092$, y una baja tendencia a la significación para la calidad general $(\mathrm{p}=, 101)$ y el valor percibido $(\mathrm{p}=, 109)$.
El modelo propuesto en la regresión lineal múltiple fue como variable dependiente las intenciones futuras de volver a realizar el servicio y como variables independientes el resto de dimensiones: valor percibido y satisfacción. La calidad general no resultó una variable predictora de las intenciones 
futuras y se omitió en una segunda prueba. La validez del modelo de regresión ha sido comprobada mediante la prueba ANOVA observable en la tabla 3, indicando que las variables independientes explican de forma significativa $(\mathrm{p} \leq, 001)$ la variable dependiente.

Tabla 3. Resumen ANOVA de la regresión lineal múltiple de predicción de las intenciones futuras respecto al valor percibido y satisfacción.

\begin{tabular}{ccccccc}
\hline & Modelo & Suma de cuadrados & gl & Media cuadrática & F & Sig. \\
\hline \multirow{2}{*}{1} & Regresión & 116,356 & 2 & 58,178 & 368,508 &, $000^{\mathrm{b}}$ \\
& Residual & 28,733 & 182 &, 158 & & \\
\hline & Total & 145,089 & 184 & & \\
\hline
\end{tabular}

a. Variable dependiente: Factor intenciones futuras

b. Variables predictoras: (Constante), Factor satisfacción, Factor Valor percibido

La tabla 4 muestra como la prueba Durbin-Watson arrojó un valor cercano a $2(1,800)$, indicando que las variables independientes, valor percibido y satisfacción, eran buenas estimadoras de la variable dependiente, satisfacción. El coe- ficiente de determinación R2 indicó que este modelo explica el $80,2 \%$ de la varianza, por tanto, el valor percibido y la satisfacción predicen en un $80,2 \%$ las intenciones futuras de los usuarios de servicios náuticos.

Tabla 4. Coeficientes del modelo y prueba de Durbin-Watson de predicción de las intenciones futuras respecto al valor percibido y satisfacción.

\begin{tabular}{cccccc}
\hline Modelo & $\mathrm{R}$ & $\mathrm{R}$ cuadrado & $\mathrm{R}$ cuadrado corregida & Error típ. de la estimación & Durbin-Watson \\
\hline 1 &, $896^{\mathrm{a}}$ &, 802 &, 800 &, 39733 & 1,800 \\
\hline
\end{tabular}

a. Variables predictoras: (Constante), Factor satisfacción, Factor Valor percibido

b. Variable dependiente: Factor intenciones futuras

Figura 1. Gráfico de probabilidad normal de los residuos tipificados de la predicción de las intenciones futuras según el valor percibido y la satisfacción.

\section{Gráfico P-P normal de regresión Residuo tipificado}

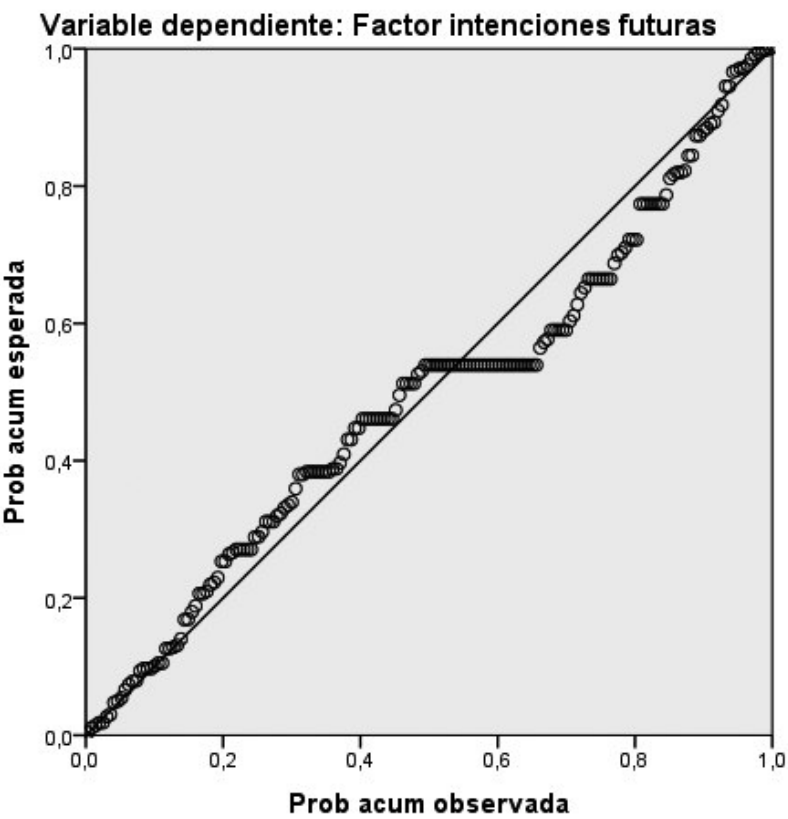

También se ha tenido en cuenta los residuos para detectar posibles desajustes dentro del modelo. La figura 1, muestra visualmente una gráfica P-P de $45^{\circ}$ indicando que el modelo propuesto cumple con el principio de homocedasticidad, al encontrarse la distribución de los valores son próximos sobre la línea de $45^{\circ}$ grados, validando el supuesto de normalidad.

El resumen del modelo de análisis de regresión lineal múltiple de los factores valor percibido y satisfacción se observa en la tabla 5, los coeficientes obtenidos por cada uno de los factores indica que la satisfacción fue el factor con más peso, con un Beta de ,732, arrojando el valor percibido una Beta de, 195 . 
Tabla 5. Coeficientes de regresión de valor percibido y satisfacción de la variable intenciones futuras.

\begin{tabular}{|c|c|c|c|c|c|c|c|c|}
\hline \multirow{2}{*}{\multicolumn{2}{|c|}{ Modelo }} & \multicolumn{2}{|c|}{ Coeficientes no estandarizados } & \multirow{2}{*}{$\begin{array}{c}\text { Coeficientes tipificados } \\
\text { Beta }\end{array}$} & \multirow{2}{*}{$\mathbf{t}$} & \multirow{2}{*}{ Sig. } & \multicolumn{2}{|c|}{ Estadísticos de colinealidac } \\
\hline & & B & Error típ. & & & & Tolerancia & FIV \\
\hline \multirow{3}{*}{1} & (Constante) &, 431 &, 173 & & 2,490 &, 014 & & \\
\hline & Factor Valor percibido & ,195 &, 047 &, 204 & 4,101 &, 000 & ,438 & 2,285 \\
\hline & Factor satisfacción &, 727 &, 050 & ,732 & 14,679 &, 000 & ,438 & 2,285 \\
\hline
\end{tabular}

\section{Discusión}

Los principales hallazgos del presente estudio muestran la relación de cada uno de los factores en total y por edades, no obteniéndose relación significativa por edades, aunque correspondiéndose el total con estudios previos como el de Theodorakis, Alexandris, Tsigilis, \& Karvounis (2013) que investigan la relación existente entre la calidad de servicio, la satisfacción del consumidor y el compromiso psicológico. Estos autores aplican el modelo de Brady, Cronin, \& Brand (2002) de un modo simplificado a la industria del fitness. Entre las principales conclusiones que obtienen se puede hacer referencia a que el entorno físico y la calidad del resultado son las dos dimensiones de calidad que tienen una influencia significativa sobre la satisfacción, siendo precisamente la satisfacción, junto con intenciones futuras las que mejores resultados arrojan a tener en cuenta como intereses de los clientes para este trabajo.

Por otra parte, determinados autores (Theodorakis, Koustelios, Robinson, \& Barlas, 2009) aplican el cuestionario QUESC de Kim y Kim (1995) en su versión de expectativas para determinar cómo pueden segmentar o diferenciar a los usuarios de instalaciones en función de variables sociodemográficas o variables como el tipo de instalación y los motivos de asistencia. Éstos por el contrario encontraron diferencias estadísticamente significativas respecto a las expectativas de calidad de servicio en función la edad; pero además también según el género, los motivos de asistencia y el tipo de instalación coincidiendo con otros trabajos realizados por García, Vegara, López \& Díaz (2016) y Aparicio, Gil, López \& Díaz (2016). Sin embargo, en relación al presente estudio, las intenciones futuras de práctica de los usuarios no relacionó con la calidad percibida, aunque sí lo hizo con valor percibido y satisfacción, siendo estos dos buenos predictores.
En esta línea obtuvieron resultados diversas investigaciones en las que la lealtad se asocia a la satisfacción como factor predictor para el consumidor de servicios deportivos (Bodet \& Bernache-Assollant, 2011). Además en relación a las actividades náuticas otros estudios establecen relación directa entre lealtad y satisfacción, pudiendo entender que las predicciones futuras de práctica pasan inexorablemente por el trabajo de estos dos factores (Segado, Roque, \& Simoes, 2016).

\section{Conclusiones}

En relación a los objetivos planteados, se puede concluir por tanto que tras la observación de las necesidades planteadas por los usuarios de servicios de chárter náutico en las islas baleares, no se presentan diferencias significativas para las cuatro dimensiones que miden la calidad del servicio percibido por los usuarios entre los tres grupos de edad establecidos; siendo el que más importancia tiene sobre los demás el de satisfacción. Además aunque las intenciones futuras de práctica por parte de estos no presenta relación con la calidad general del servicio, si mantiene una importante relación con lo satisfechos que quedaron del servicio y el valor percibido.

Con todo esto se observa como la investigación sobre la calidad de servicio en entidades y servicios deportivos náuticos se ha centrado en la evaluación de expectativas y percepciones de los usuarios para determinar los atributos, que desde el punto de vista del usuario, configuran la percepción de calidad en los servicios. Este trabajo ha pretendido servir como punto de partida para profundizar en este conocimiento analizando la calidad de servicio de empresas de chárter náutico debido a la escasez que para los mismos se encuentra en la bibliografía.

\section{Bibliografía}

1. Angosto, S. (2014). Diseño y validación de un cuestionario sobre calidad percibida en eventos deportivos populares (CAPPEP). Trabajo de Fin de Máster. Murcia: Universidad de Murcia.

2. Aparicio Sarmiento, A., Gil López, M. I., López Sánchez, G. F. \& Díaz Suárez, A. (2016). Satisfacción de usuarios de dos clubes de pádel de Cartagena (Región de Murcia). SPORT TK: Revista EuroAmericana de Ciencias del Deporte, 5(2), 27-32.

3. Bodet, G., \& Bernache-Assollant, I. (2011). Consumer Loyalty in Sport Spectatorship Services: The Relationships with Consumer Sa-

4. Brady, M. K., Cronin, J. J., \& Brand, R. R. (2002). Performance-only measurement of service quality: a replication and extension. Journal of Business Research, 55(1), 17-31.

5. Calabuig-Moreno, F., Crespo-Hervas, J., Prado-Gasco, V., MundinaGomez, J., Valantine, I., \& Stanislovaitis, A. (2016). Quality of sporting events: validation of the eventqual scale. Transformations in Business \& Economics, 15(2), 21-32. 
6. Crespo, J. (2011). Los eventos deportivos: Calidad de servicio, valor percibido y satisfacción de los espectadores. Tesis doctoral no publicada. Valencia: Universidad de Valencia.

7. Favro, S., Grzetic, Z., \& Kovacic, M. (2010). Towards sustainable yachting in croatian traditional island ports. Environmental Engineering and Management Journal, 9(6), 787-794.

8. Gallarza, M. \& Gil, I. (2006b]. Value dimensions, perceived value, satisfaction and loyalty: an investigation of university students' travel behaviour. Tourism Management, 27(3), 437-452.

9. García Mayor, J., Vegara Ferri, J. M., López Sánchez, G. F., Díaz Suárez A. (2016). Satisfacción de usuarios de servicios deportivos en Orihuela (Alicante). SPORT TK: Revista EuroAmericana de Ciencias del Deporte, 5(Supl.), 155-162.

10. Getz, D. (2008). Event tourism: Definition, evolution, and research. Tourism Management, 29(3), 403-428.

11. Hightower Jr, R., Brady, M. K., \& Baker, T. L. (2002). Investigating the role of the physical environment in hedonic service consumption: an exploratory study of sporting events. Journal of Business Research, 55(9), 697-707.

12. Jovanovic, T., Dragin, A., Armenski, T., Pavic, D., \& Davidovic, N. (2013). What demotivates the tourist? Constraining factors of nautical tourism. Journal of Travel \& Tourism Marketing, 30(8), 858-872.

13. Kim, D., \& Kim, S.Y. (1995). QUESC: An instrument for assesing the service quality of sport centers in Korea. Journal of Sport Management, 9(2), 208-220.

14. Kovacic, M. (2010). Selecting the location of a nautical toursim port by applying promethee and gaia methods case study-croatian northern adriatic. Promet-Traffic \& Transportation, 22(5), 341-351.

15. Lepp, A., \& Gibson, H. (2008). Sensation seeking and tourism: Tourist role, perception of risk and destination choice. Tourism Management, 29(4), 740-750.

16. López-Sánchez, G. F., Díaz-Suárez, A., Radzimiński, Ł., \& Jastrzębski, Z. (2017). Effects of a 12-week-long program of vigorous-intensity physical activity on the body composition of 10-and 11-year-old children. Journal of Human Sport and Exercise, 12(1), 235-244. doi:10.14198/ jhse.2017.121.19

17. Mikulic, J., Kresic, D., \& Kozic, I. (2015). Critical factors of the maritime yachting tourism experience: an impact asymmetry analysis of principal components. Journal of Travel \& Tourism Marketing, 32, S30-S41.

18. Moon, K. S., Kim, M., Ko, Y. J., Connaughton, D. P., \& Lee, J. H.
(2011). The influence of consumer's event quality perception on destination image. Managing Service Quality, 21(3), 287-303.

19. Oliver, R. (1981]: "Measurement and Evaluation of Satisfaction Processes in Retail Settings". Journal of Retailing, 57(3), 25-46.

20. Parasuraman, A., Zeithaml, V. A., \& Berry, L. L. (1985). A conceptialmodel of service quality and its implications for future research. Journal of Marketing, 49(4), 41-50.

21. Parasuraman, A., Zeithaml, V. A., \& Berry, L. L. (1988). Sevqual amultiple-item scale for measuring consumer perceptions of service quality. Journal of Retailing, 64(1), 12-40.

22. Robinot, E., \& Giannelloni, J. L. (2010). Do hotels' "green” attributes contribute to customer satisfaction? Journal of Services Marketing, 24(23), 157-169

23. Segado, F. S., Roque, J. I. A., \& Simoes, M. C. R. (2016). Relation among satisfaction and loyalty of the users of nautical camps. Revista Internacional de Medicina y Ciencias de la Actividad Fisica y del Deporte, 16(61), 17-32.

24. Shonk, D. J., \& Chelladurai, P. (2008). Service quality, satisfaction, and intent to return in event sport tourism. Journal of Sport Management, 22(5), 587-602.

25. Theodorakis, N. D., Alexandris, K., Tsigilis, N., \& Karvounis, S. (2013). Predicting spectators' behavioural intentions in professional football: The role of satisfaction and service quality. Sport Management Review, 16(1), 85-96.

26. Theodorakis, N. D., Koustelios, A., Robinson, L., \& Barlas, A. (2009) Moderating role of team identification on the relationship between service quality and repurchase intentions among spectators of professional sports. Managing Service Quality, 19(4), 456-473.

27. Vespalec, T., Pavlík, J., Zvonař, M. \& Zeman, T. (2016). Physical activity of Czech schoolchildren in the autumn season. Journal of Human Sport and Exercise, 11(Proc1), S137-S145. doi:10.14198/jhse.2016.11. Proc1.04

28. Wang, N. (1999). Rethinking authenticity in tourism experience. Annals of Tourism Research, 26(2), 349-370.

29. Yepes, V., \& Medina, J. R. (2005). Land Use Tourism Models in Spanish Coastal Areas. A Case Study of the Valencia Region. Journal of Coastal Research, 83-88.

30. Zeithaml, V. A., Berry, L. L., \& Parasuraman, A. (1996). The behavioral consequences of service quality. Journal of Marketing, 60, 31-46. 